\title{
Effects of Diabetic Hyperglycemia on Central Ang-(1-7)-Mas-R-nNOS Pathways in Spontaneously Hypertensive Rats
}

\author{
He Li Xian Liu Zhongqiao Ren Jinxia Gu Yingjie Lu Xiaoyun Wang \\ Lan Zhang \\ Department of Cardiology, The Fourth Clinical Hospital of Harbin Medical University, Harbin, China
}

\section{Key Words}

Diabetic $\cdot$ Hypertension $•$ Angiotensin-(1-7) Oxidative stress $•$ Brain natriuretic peptide

\begin{abstract}
Background/Aims: Hypertension is a major cause of stroke, and diabetes can increase incidence of this disease. We determined the role played by central angiotensin-(1-7) [Ang(1-7)] pathway in modulating spontaneously hypertension with diabetic hyperglycemia. Methods: Western Blot analysis and ELISA were used to determine the protein expression of Ang-(1-7) and its signal pathway Mas-R-nNOS in the cerebral cortex and hippocampus of spontaneously hypertensive rats (SHR) and control animals. In a subset of animals, diabetic hyperglycemia was induced by systemic injection of streptozotocin (STZ). We analyzed a relationship between the levels of central Ang-(1-7) and plasma brain natriuretic peptide (BNP) indicating a risk of ischemic stroke. We further examined the effects of Ang-(1-7) on arterial blood pressure. Results: Our findings demonstrated for the first time that administration of STZ 1) attenuates the levels of Ang-(1-7) in the cerebral cortex and hippocampus, which are closely linked to plasma BNP; and 2) leads to downregulation of central Ang-(1-7)-Mas-RnNOS pathways. Notably, STZ has greater effects in SHR. Additionally, inhibition of oxidative stress can largely improve downregulation of Ang-(1-7) in diabetic SHR. Moreover, central stimulation of Ang-(1-7) pathway or a blockade of oxidative stress improves systolic blood pressure in diabetic SHR. Conclusions: The Ang-(1-7) signaling pathway is engaged in the adaptive mechanisms associated with diabetic hypertension, suggesting that enhancing Ang-(1-7)-Mas-R-nNOS system is likely to be beneficial in preventing against cardiovascular and cerebrovascular dysfunction and vulnerability related to spontaneously hypertension, particularly to diabetic hypertension.
\end{abstract}

\section{Introduction}

Diabetes broadly leads to increased incidence of cardiovascular and cerebrovascular diseases. It has been reported that hypertensive patients with diabetes have a greater risk 
to suffer intracerebral hemorrhage, and they have the worse outcomes than hypertensive patients without diabetes [1]. In a general agreement, hyperglycemia is a major underlying cause of stroke in patients with hypertension; whereas hypertension is one of the most common causes of stroke in clinics [1]. Animal studies further suggest that multiple pathways such as oxidative stress and inflammation are likely involved in the worsened deficits in diabetes after cerebrovascular diseases [2-5]. Nevertheless, the underlying mechanisms by which pathophysiological development of diabetic dysfunction contributes to those diseases need to be clarified.

Angiotensin-converting enzyme 2 (ACE2) has been identified to directly cleave angiotensin (Ang II) to Ang-(1-7) and the G-protein coupled receptor Mas-R is recognized as the first binding site for Ang-(1-7) [6-8]. The presence of Mas-R has initially been recognized in the cortex and hippocampus of rat brain [9], and afterward studies demonstrate that Mas- $R$ is expressed in diverse regions of the brain including some areas related to cardiovascular regulation $[10-13,14]$. A number of studies have demonstrated that this peptide is involved in cardiovascular and cerebrovascular actions. It is noted that opposed to Ang II, the effects of Ang-(1-7) are primarily beneficial via counter-regulating Ang II actions [15]. The role for Ang-(1-7) in central regulation of cardiovascular and sympathetic nervous activities and in the pathogenesis of neurogenic hypertension has been reported [16, 17]. Importantly, recent reports show that intracerebroventricular (ICV) injection of Ang-(1-7) or central over-expression of ACE2 has beneficial effects on ischemic stroke [18, 19]. Also, evidence shows that Ang-(1-7) exerts its actions via a NO dependent mechanism [20,21].

Given that Ang-(1-7) pathway plays an important role in protecting neuronal tissues from ischemic cardiovascular and cerebrovascular injuries [22], in the current study we examined the levels of Ang-(1-7) and protein expression of Mas-R-nNOS pathway in the cerebral cortex and hippocampus of normotensive rats and spontaneously hypertensive rats (SHR) following administration of streptozotocin (STZ). The cortex and hippocampus have been selected in this study since Mas- $\mathrm{R}$ is expressed in those brain regions $[9,11]$ and they are linked to improvement of Ang-(1-7) in neurological deficits and stroke outcome induced by cerebral ischemic insult [23]. The role played by central Ang-(1-7) system in modulating arterial blood pressure (BP) was further determined. Also, we analyzed a relationship between the levels of central Ang-(1-7) and plasma brain natriuretic peptide [BNP, or termed B-type natriuretic peptide 45 (BNP-45) in rats], as an indicator of ischemic stroke risk. In addition, engagement of oxidative stress was examined after antioxidant tempol, a mimetic of superoxide dismutases (SOD), was systemically administered. Overall, our hypotheses included that 1) the Ang-(1-7) signal pathway and oxidative stress contribute to the adaptive mechanisms involved in diabetic hypertension; and 2) stimulation of central Ang-(1-7)Mas-R-nNOS system or inhibition of oxidative stress improves hypertension in STZ rats and thereby has beneficial effects on cardiovascular and cerebrovascular dysfunction in diabetic hypertension.

\section{Materials and Methods}

\section{Animals}

All the animal procedures were approved by the Institutional Animal Care \& Use Committee of Harbin Medical University, which were in compliance with the Guideline for the Care and Use of laboratory Animals of the U.S. National Health Institute. Male Wistar-Kyoto (WKY) and SHR rats (12 weeks old) were used in our experiments.

\section{Induction of diabetes}

Streptozotocin (STZ) was freshly dissolved in $0.9 \%$ sterile saline and diabetes was induced by a single injection of STZ (70 mg/kg i.p., Sigma Co.) as described previously [24]. Diabetes was confirmed by measurements of blood glucose concentrations in samples obtained from the tail vein 4 weeks after injection of STZ. Rats with blood glucose concentration $>350 \mathrm{mg} / \mathrm{dl}$ were included in the study. Age- and 


\section{Cellular Physiology Cell Physiol Biochem 2016;40:1186-1197 \begin{tabular}{l|l|l} 
and Biochemistry Published $10.1159 / 000453172$ & (c) 2016 The Author(s). Published by S. Karger AG, Basel \\
www.karger.com/cpb
\end{tabular} \\ Li et al.: Central Ang 1-7 and Diabetic Hypertension}

Fig. 1. A schematic diagram showing the experimental protocols in the study.

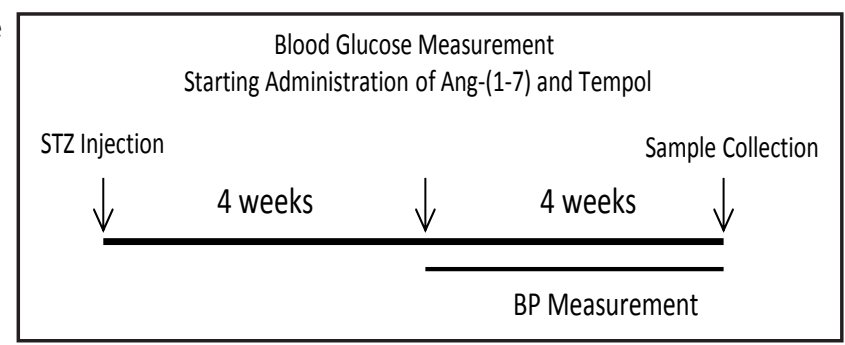

Blood Glucose Measurement

body weight-matched rats with saline injection were used as controls of non-diabetes. Thus, in this report, the rats were divided into four groups as: WKY control; WKY with STZ; SHR control and SHR with STZ, respectively. In an additional experiment, Ang-(1-7) (75 $\mathrm{gg} / \mathrm{kg}$ twice a day) and A779 (150 $\mu \mathrm{g}$ daily) were given via ICV injection $[23,25]$ and tempol $(50 \mathrm{mg} / \mathrm{kg}$ i.p. daily) was given for 4 weeks. At the end of all interventions, the rats were 20 weeks old. Arterial BP and heart rate (HR) were measured weekly by the tail-cuff method using an animal blood pressure analyzer before brain tissues were removed. Fig. 1 shows the experimental protocols.

In order to perform ICV injection, animals were cannulated with a stainless steel cannula aimed at the right lateral ventricle (coordinates: $3.7 \mathrm{~mm}$ posterior to the bregma, $4.1 \mathrm{~mm}$ lateral to the midline, and 3.5 $\mathrm{mm}$ under the dura). The guide cannula was fixed to the skull using dental zinc cement and jewelers' screw. The cannula was then connected to a $20 \mu \mathrm{l}$ of Hamilton syringe with polycarbonate tubing when Ang-(1-7) and A779 were injected. Each drug was suspended in $5 \mu \mathrm{l}$ artificial cerebrospinal fluid and was injected over 1 minute.

ELISA

The levels of Ang-(1-7) in the cerebral cortex and the hippocampal CA1 region were measured using an Ang-(1-7) Competitive ELISA kit following the manufacturer's instructions (Cat\# ABIN512915, Antibodiesonline Inc., Atlanta, GA). Briefly, the diluted tissue supernatant (100 $\mu \mathrm{l})$ was placed in a 96-well goat antirat IgG-coated plate and incubated for 2 hours. After incubation, the plate was washed using the provided washing buffer, and the color was developed by adding substrate (200 $\mu \mathrm{l})$ after $45 \mathrm{~min}$ and determined by an ELISA plate reader. The amount of Ang-(1-7) was calculated using a standard curve. A similar method was also used to examine the levels of 8-iso PGF $2 \alpha$ in the cerebral cortex and the hippocampal CA1 region [commercially available enzyme immunoassay kit [8-isoprostaglandin F2 $\alpha$ (8-iso PGF2 $\alpha$ ), Cayman Chemical Co.].

BNP-45 levels were determined by Rat BNP-45 Sandwich ELISA Kit (Product\#ab108816, Abcam Co., Cambridge, MA). Briefly, standard or plasma samples were added to each well. The wells were incubated at room temperature and then aspirated and washed. A biotinylated BNP-45 antibody was added to each well and incubated, followed by washes. Then, streptavidin-peroxidase conjugate was added and incubated and washed. Afterward, chromogen substrate solution was added to each well and incubated and the reaction was stopped by adding stop solution. Then, the optical density was detected immediately using a microplate reader.

\section{Western blot analysis}

To examine expression of Mas-R and nNOS, the cortex and hippocampal tissues were processed using a standard Western Blot procedure. Briefly, total protein was extracted by homogenizing the cerebral cortex and hippocampal samples in ice-cold immunoprecipitation assay buffer. The lysates were centrifuged and the supernatants were collected for measurements of protein concentrations using a BCA reagent (Pierce BCA Protein Assay Kit Cat\#23225, Life Technologies, Grand Island, NY). After being denatured by heating at $95^{\circ} \mathrm{C}$ for $5 \mathrm{~min}$ in buffer, the supernatant samples ( 2 samples for each experimental group) containing $20 \mu \mathrm{g}$ of protein were loaded onto 4-20\% Mini-PROTEAN TGX gels and electrically transferred to a polyvinylidene fluoride membrane. The membrane was blocked in 5\% nonfat milk in 0.1\% Tween-TBS buffer and was incubated overnight with primary antibodies (rabbit anti-Mas-R at 1: 200, Alomone Labs Cat\# AAR-013; and rabbit anti-nNOS at 1:500, Cayman Chemical Co. Cat\#160870). Next, the membranes were washed and incubated with an alkaline phosphatase conjugated anti-rabbit secondary antibody (1:1000). The immunoreactive proteins were detected by enhanced chemiluminescence system (Cell Signaling Technology, 
Beverly, MA). The bands recognized by the primary antibody were visualized by exposure of the membrane onto an x-ray film. The membrane was stripped and incubated with mouse anti- $\beta$-actin to show equal loading of the protein. Then, the film was scanned and the optical density of Mas-R and nNOS as well as $\beta$-actin bands was analyzed using the Scion Image software. The values for densities of Mas-R and nNOS immunoreactive bands $/ \beta$-actin band from the same lane were determined. Each of the values was then normalized to a control sample.

\section{Statistical analysis}

All the data were analyzed using a two-way repeated-measure ANOVA. Values are presented as means \pm SEM. For all analyses, differences were considered significant at $P<0.05$. All statistical analyses were performed by using SPSS for Windows version 11.0.

\section{Results}

\section{General measurements}

The rats developed hyperglycemia 4 weeks after STZ injection. Also, an increase of body weight was significantly attenuated in STZ rats $(P<0.05 \mathrm{vs}$. non-STZ control rats with saline) as compared with non-STZ rats (Table 1). Systolic blood pressure (SBP) was higher in SHR than in WKY rats (Fig. 2). There was no significant difference in HR between SHR and WKY rats (Table 1).

Table 1. General Measurements. SBP: systolic blood pressure; HR: heart rate. ${ }^{*} P<0.05$ vs. WKY rats; ${ }^{* *} P<$ 0.05 vs. SHR. There were no significant differences in body, glucose and HR observed between SHR+STZ and SHR+STZ with administration of Ang-(1-7) and tempol. Note that all measurements were taken at the end week of experiments

\begin{tabular}{lcccccr}
\hline Rats & WKY & WKY+STZ & SHR & SHR+STZ & $\begin{array}{c}\text { SHR+STZ } \\
+ \text { Ang-(1-7)/A779 }\end{array}$ & $\begin{array}{c}\text { SHR+STZ } \\
\text { +Tempol }\end{array}$ \\
\hline Number of rats & 20 & 25 & 22 & 24 & 15 & 12 \\
Body weight (g) & $425 \pm 15$ & $356 \pm 14^{*}$ & $395 \pm 16$ & $318 \pm 15^{* *}$ & $325 \pm 16$ & $322 \pm 17$ \\
Blood glucose (mg/dl) & $125 \pm 10$ & $485 \pm 16^{*}$ & $135 \pm 8$ & $482 \pm 10^{* *}$ & $479 \pm 15$ & $480 \pm 18$ \\
HR (beats/min) & $395 \pm 8$ & $390 \pm 9$ & $408 \pm 8$ & $405 \pm 7$ & $400 \pm 12$ & $397 \pm 12$ \\
\hline
\end{tabular}

Fig. 2. (A): Ang-(1-7) injected into the brain decreased SBP in diabetic SHR rat and the effects were inhibited by A779. Also, tempol decreased SBP in diabetic SHR rats. The effects of both Ang(1-7) and tempol began 3 weeks after their administration. ${ }^{*} P<0.05$, diabetic SHR with Ang-(1-7) $(n=8)$ and diabetic SHR with tempol $(\mathrm{n}=12) v s$. diabetic SHR rats without

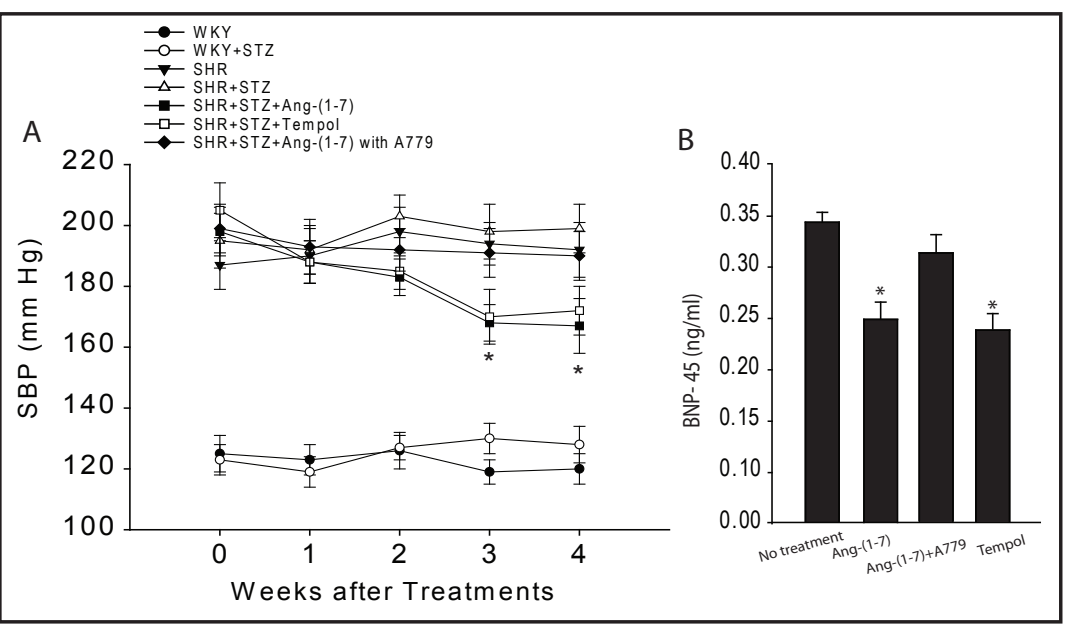
treatments ( $\mathrm{n}=15) ;{ }^{*} P<0.05$, also indicated Ang-(1-7) injection $v s$. Ang-(1-7) with using A779 (n=7). (B): The levels of plasma BNP-45 were significantly attenuated in diabetic SHR rats after using of Ang-(1-7) $(\mathrm{n}=8)$ and tempol $(\mathrm{n}=12)$, respectively. ${ }^{*} P<0.05$ vs. diabetic SHR rats with no treatment $(\mathrm{n}=15)$ and diabetic SHR rats given with Ang-(1-7) and A779 (n=7).

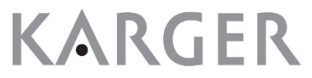


Fig. 3. The levels of Ang-(1-7) in the cerebral cortex (A) and the hippocampal CA1 (B) were significantly decreased in WKY $(n=15)$ and SHR rats $(n=16)$ after STZ injection. The effects were greater in SHR rats. Ang-(1-7) also appeared to be smaller in SHR rats $(n=10)$ than in WKY rats $(\mathrm{n}=8) .{ }^{*} P<0.05$ vs. respective WKY and SHR control rats without STZ. $\dagger P<0.05$, indicated SHR with STZ vs. WKY with STZ. \# $P<0.05$, indicated SHR vs. WKY without STZ.
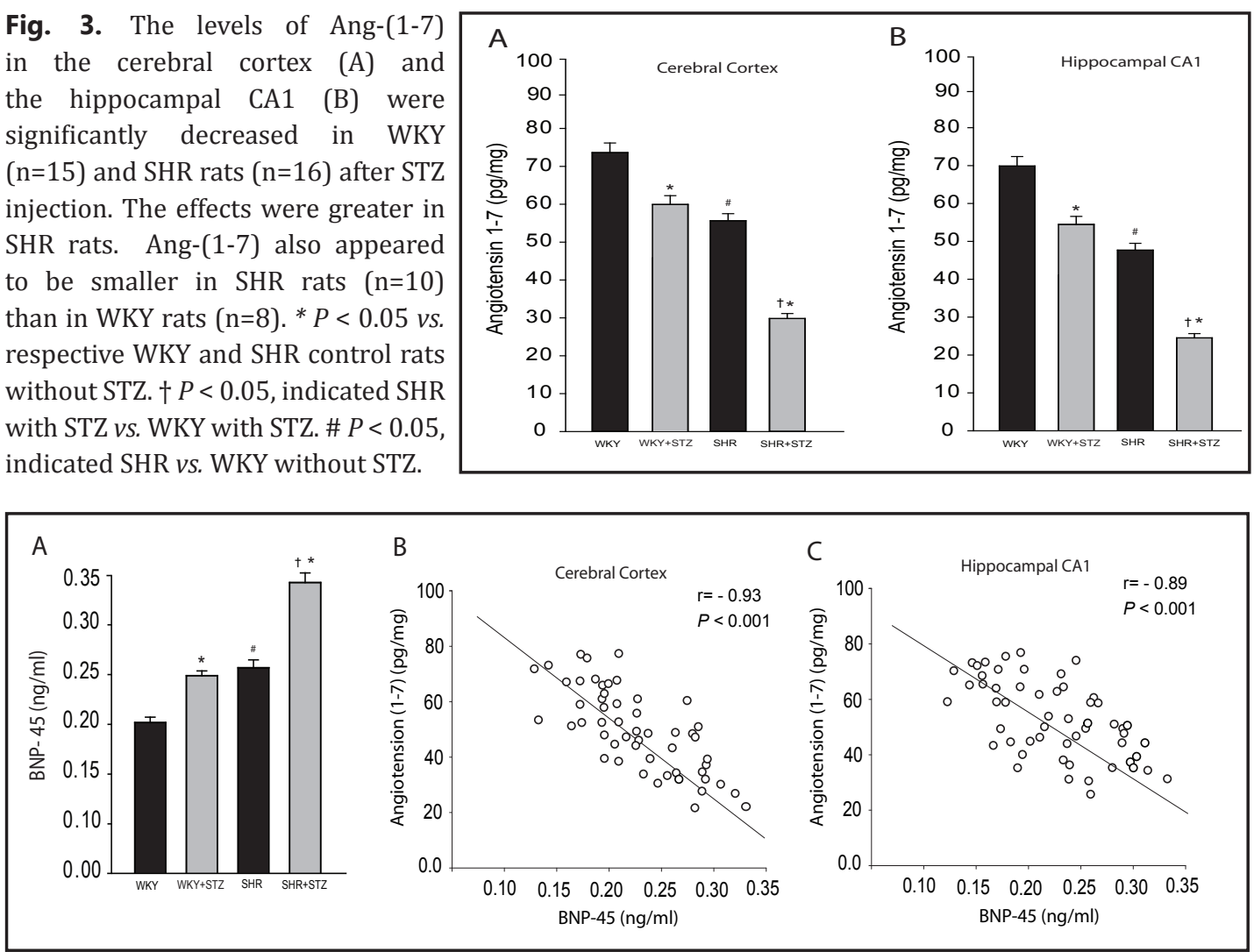

Fig. 4. (A): Plasma levels of BNP-45 were significantly increased in WKY $(n=15)$ and SHR $(n=16)$ after STZ. There was a greater level of BNP-45 in SHR rats $(n=10)$ than that in WKY rats $(n=8)$. Also, STZ increased BNP-45 to a greater degree in SHR rats as compared with WKY rats. ${ }^{*} P<0.05$ vs. WKY and SHR controls. $\dagger P$ $<0.05$, indicated SHR with STZ vs. WKY with STZ. \# P<0.05, indicated SHR vs. WKY without STZ. (B) \& (C): showing that there was an inverse relation between plasma BNP-45 and Ang-(1-7) in the cerebral cortex and in the hippocampal CA1 region, respectively.

Levels of Ang-(1-7)

The levels of Ang-(1-7) in the cerebral cortex and hippocampus CA1 region were measured and shown in Fig. 3A\&B. Ang-(1-7) was significantly decreased in the cerebral cortex and hippocampus CA1 region of WKY and SHR rats after STZ injection. A lower level of Ang-(1-7) was observed in those brain areas in SHR rats than that in WKY rats. Our result further shows that STZ decreased Ang-(1-7) to a greater degree in SHR rats. i.e., Ang-(1-7) was decreased by $62 \%$ in the cortex and 59\% in CA1 region in SHR rats with STZ $(P<0.05$ vs. WKY rats); and Ang-(1-7) was decreased by 35\% in the cortex and 38\% in CA1 region in WKY rats with STZ.

\section{A relationship between Ang-(1-7) and BNP-45}

We further examined plasma BNP-45 (Fig. 4A) and determined a relationship between Ang-(1-7) and BNP-45 (Fig. 4B\&C). Plasma BNP-45 was increased in WKY and SHR rats with injection of STZ. A greater level of BNP-45 in SHR rats was observed as compared with WKY rats. Also, STZ increased BNP-45 to a greater degree in SHR rats as compared with WKY rats (by $32 \%$ in WKY; and 45\% in SHR; $P<0.05$ vs. WKY). Furthermore, a liner relationship analysis was performed, demonstrating that there was an inverse relation between plasma BNP-45 and Ang-(1-7) in the cerebral cortex $(r=-0.93, P<0.001)$ and in the hippocampal CA1 region $(\mathrm{r}=-0.89, P<0.001)$. 
Fig. 5. STZ significantly attenuated Mas-R receptors and nNOS. (A): Top panels show representative bands of Mas- $\mathrm{R}$ expression in four groups of rats; and bottom panels show averaged data in the cerebral cortex (left) and in the hippocampal CA1 region (right). (B): Top panels: representative bands of nNOS expression in four groups; and bottom panels: averaged data in the cerebral cortex (left panel) and in the hippocampal CA1 area (right panel). ${ }^{*} P<$ 0.05 vs. respective WKY $(n=12)$ and SHR control rats $(\mathrm{n}=12)$ without STZ. $\dagger P<0.05$, indicated SHR with STZ $(\mathrm{n}=16) v s$. WKY with STZ $(\mathrm{n}=15)$. \# $P<$ 0.05 , indicated SHR vs. WKY without STZ.

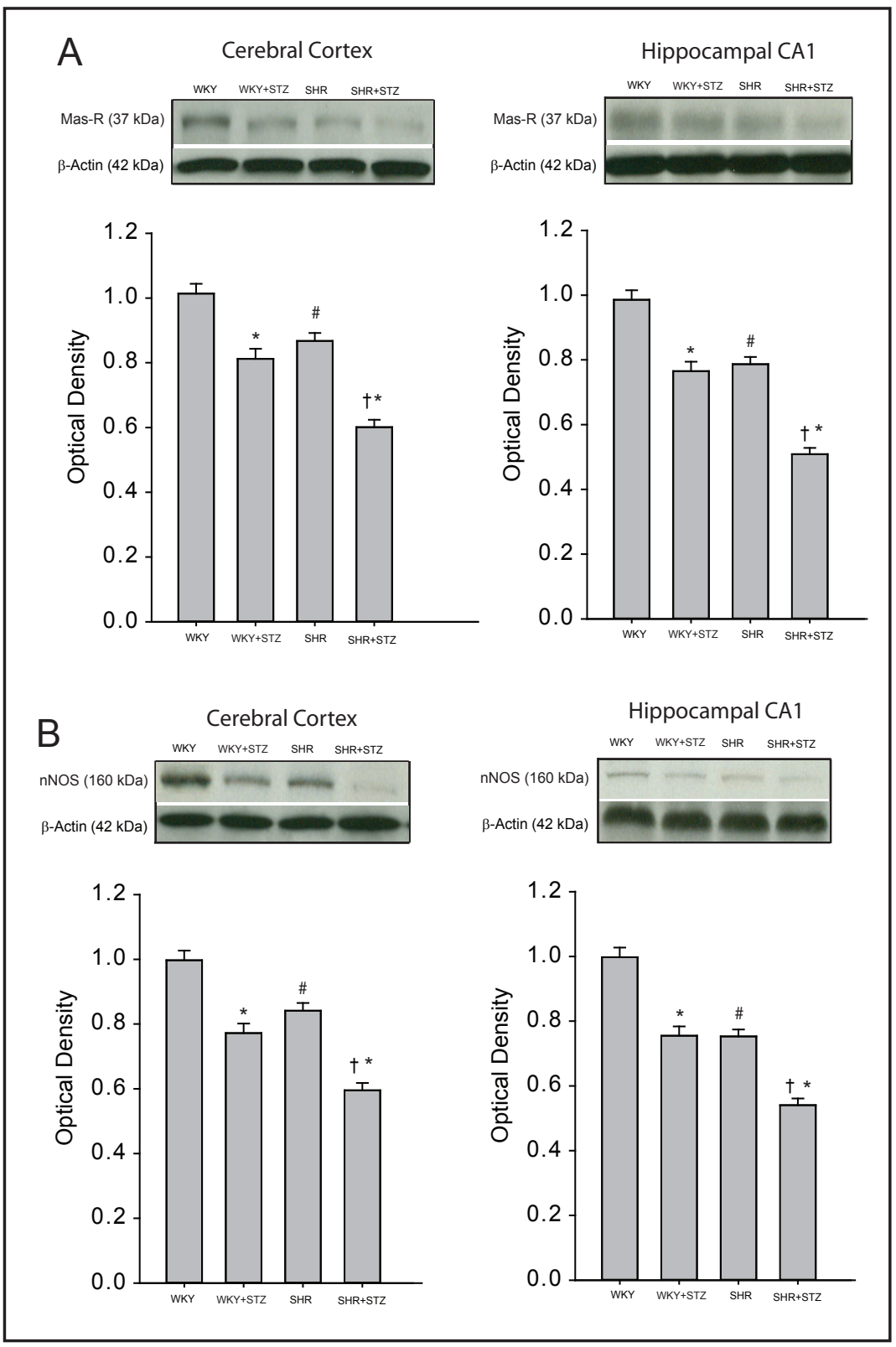

Expression of Mas-R and nNOS pathways

In this study, the protein levels of Mas-R receptors and nNOS in the cerebral cortex and the hippocampal CA1 region of WKY and SHR rats were examined after injection of STZ (Fig. $5 A \& B)$. Administration of STZ significantly attenuated expression of Mas-R and nNOS in the cerebral cortex and the hippocampal CA1 region $(P<0.05$, vs. with saline injection as control in both WKY and SHR rats). Nonetheless, the effects of STZ appeared to be larger in SHR rats than in WKR rats. Also, Mas-R and nNOS pathways in the cerebral cortex and the hippocampal CA1 region were downregulated in SHR rats as compared with WKY.

\section{Effects of oxidative stress}

In a subgroup of experiments, we examined the role played by oxidative stress in regulating expression of Ang-(1-7) (Fig. 6). First, we measured concentrations of 8-iso PGF $2 \alpha$ as an index of oxidative stress in the cerebral cortex and hippocampus CA1 region. Our results show that the levels of 8-iso PGF2 $\alpha$ were significantly increased in the cerebral cortex and hippocampus CA1 region of WKY and SHR rats with injection of STZ (Fig. 6A). 
Fig. 6. (A): Concentrations of 8-iso PGF2 $\alpha$ were significantly increased in the cerebral cortex and hippocampus CA1 region of WKY and SHR rats with injection of STZ. STZ induced a greater increase in 8-iso PGF2 $\alpha$ in those brain areas of SHR rats. Note that administration of tempol attenuated amplified 8-iso PGF2 $\alpha$ in WKY rats with STZ and SHR rats. ${ }^{*} P<0.05$ vs. respective WKY and $(\mathrm{n}=12)$ SHR control rats $(\mathrm{n}=12)$ without STZ. $\dagger P<0.05$, indicated SHR with STZ $(\mathrm{n}=16)$ vs. WKY with STZ ( $\mathrm{n}=15)$. \# $P<0.05$, indicated SHR vs. WKY without STZ. ** $P<0.05$ $v s$. respective group with no tempol. (B): demonstrating that there was an inverse relation between 8-iso PGF2 $\alpha$ and Ang-(1-7) in the cerebral cortex and in the hippocampal

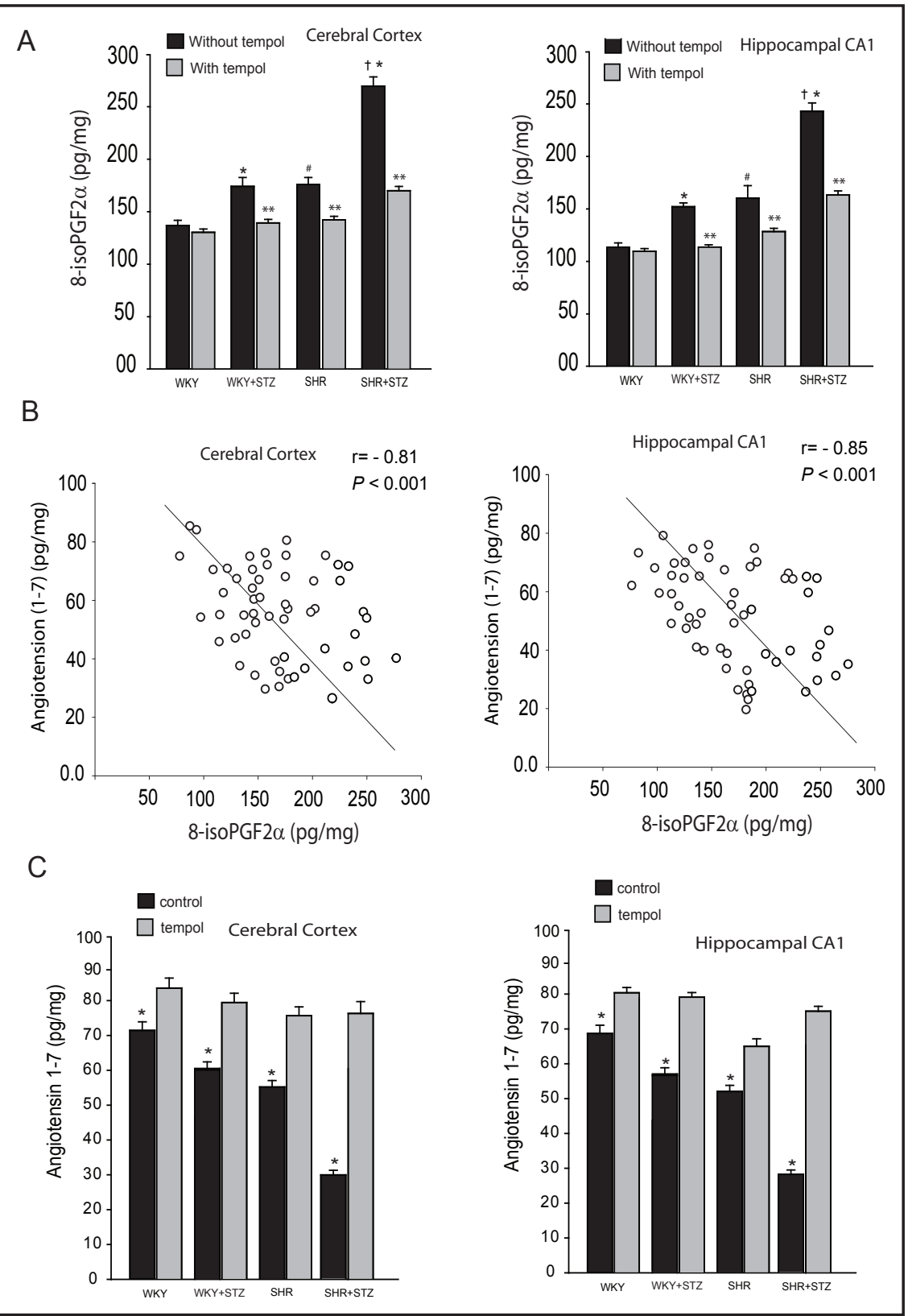

CA1 region. (C): showing that tempol restored downregulation of Ang-(1-7) in the cerebral cortex and hippocampus CA1. Tempol had greater effects on the levels of Ang-(1-7) in the cerebral cortex and hippocampus CA1 region of SHR rats with STZ. * $P<0.05 \mathrm{vs}$. tempol injection. The number of animals with tempol administration = 8 in WKY; 10 in WKY+STZ; 10 in SHR and 8 in SHR+STZ.

Note that STZ induced a greater increase in 8-iso PGF2 $\alpha$ in those brain areas of SHR rats. Administration of tempol attenuated amplification of 8-iso PGF $2 \alpha$ in these animals. Our results further demonstrate that there was an inverse relation between 8-iso PGF2 $\alpha$ and Ang-(1-7) in the cerebral cortex $(\mathrm{r}=-081, P<0.001)$ and in the hippocampal CA1 region $(\mathrm{r}=$ $-0.85, P<0.001$ ) (Fig. 6B).

Moreover, as tempol was given to attenuate 8 -iso $\mathrm{PGF} 2 \alpha$, this largely restored decreases in Ang-(1-7) of the cerebral cortex and hippocampus CA1 (Fig. 6C). Note that tempol significantly attenuated the levels of 8-iso PGF2 $\alpha$ in four groups of animals. Nonetheless, tempol had greater effects on the levels of Ang-(1-7) in the cerebral cortex and hippocampus CA1 region of SHR rats with STZ as compare with other groups. 


\section{Cellular Physiology Cell Physiol Biochem 2016;40:1186-1197 \begin{tabular}{l|l} 
and Biochemistry & DOI: 10.1159/000453172 \\
Published online: 2016 The Author(s). Published by S. Karger AG, Basel \\
www.karger.com/cpb
\end{tabular} \\ Li et al.: Central Ang 1-7 and Diabetic Hypertension}

\section{Effects of Ang-(1-7) and tempol on BP and HR}

In order to determine the beneficial role of Ang-(1-7) in arterial BP of diabetic hypertension, SBP was examined after Ang-(1-7) was given into the brain of diabetic SHR rats via ICV injection (Fig. 2A). Three weeks after injection of Ang-(1-7), SBP was significantly reduced in SHR with STZ. With using A779 blocking Mas-R, the effects of Ang-(1-7) were inhibited. Nevertheless, this failed to significantly alter HR and the levels of blood glucose (Table 1). Likewise, tempol also decreased SBP in diabetic SHR rats without changing HR and the levels of blood glucose (Fig. 2A and Table 1). At the end of experiments, the levels of plasma BNP-45 were also examined and Fig. 2B shows that the levels of BNP-45 were decreased in diabetic SHR animals after administration of Ang-(1-7) and tempol.

In additional group ( $\mathrm{n}=10)$, we examined SBP after ICV injection of Ang-(1-7) in nondiabetic SHR rats. Ang-(1-7) did not significantly alter SBP in non-diabetic SHR animals. i.e., SBP ( $\mathrm{mm} \mathrm{Hg}$ ) was $203 \pm 10$ (before injection; $P>0.05 \mathrm{vs}$. all time points after injection), $197 \pm 9$ (1 week), 195 \pm 8 ( 2 weeks), 196 \pm 10 (3 weeks) and 193 \pm 12 (4 weeks) following injection of Ang-(1-7).

\section{Discussion}

Although diabetes is associated with increased cardiovascular morbidity and mortality, prior reports have also demonstrated that diabetic patients without metabolic syndrome do not have greater prevalence of coronary arterial disease [26, 27]. In addition, metaanalysis of major large clinical trials fail to show a clear benefit of intensive glucose control on all-cause mortality, except for the reduction in cardiovascular risk [28]. This is likely due to confounding factors, such as obesity and hyperinsulinemia. Nevertheless, the causeeffect relationship of hyperglycemia and cardiovascular disease is yet to be established. Spontaneously hypertensive STZ-induced diabetic (SHR/STZ) rats are generally used to study the mechanisms involved in hypertension with diabetes [29] as they develop significant elevations in plasma glucose levels, polyuria, albuminuria, and glycosuria and a pronounced loss of abdominal adipose tissue and body weight together with severe hypertension [30]. Thus, this model was employed in our present study. The main findings of the present study are that 1) the levels of Ang-(1-7) and Ang-(1-7)-Mas-R-nNOS pathways are impaired in the cerebral cortex and hippocampus after STZ; 2) the effects of STZ appear to be greater in SHR; 3) central administration of Ang-(1-7) decreases SBP in diabetic SHR animals; 4) inhibition of oxidative stress significantly improves the worsen Ang-(1-7) and decreases SBP in diabetic SHR.

Ang-(1-7) activated-Mas- $R$ receptor has various physiological functions such as regulating blood pressure, attenuating the progress of atherosclerosis through inhibiting vascular smooth muscle-cell proliferation and restoring endothelial function [31-33]. The protective role of the Ang-(1-7)-Mas-R pathways in cardiovascular and cerebrovascular diseases has gained much attention for the last several years [34, 35]. Activation of Ang(1-7)-Mas-R signaling attenuates the development of hypertension and the pathologic progress of atherosclerosis $[17,36]$. A recent study has shown that neural over-expression of ACE2 lowers neurological deficit scores and leads to smaller stroke volumes following middle carotid artery occlusion-induced stroke in mice [19], suggesting that ACE2 protects the brain from ischemic injury. Also, this study suggests that activation of ACE2/Ang-(1-7)Mas- $\mathrm{R}$ pathways can exert a direct neuroprotective action by alleviating ischemia-induced cell swelling and cell death [19]. In addition, ICV infusion of Ang-(1-7) or an ACE2 activator reduces ischemic injury in rats that are caused by a reduction in cerebral blood flow $[18,31]$. A neuroprotective effect of central administration of Ang-(1-7) on ischemic injury is through inhibiting the NF-kappa B inflammation pathway [31]. In the present study, we found that Ang-(1-7)-Mas-R-nNOS pathways are impaired in either SHR rats or STZ animals and this appears to be worsened in SHR after injection of STZ. 
The levels of plasma BNP have been considered as indication for screening, diagnostic, prognostic, treatment and monitoring treatment of patients with cerebrovascular diseases $[37,38]$, because BNP driven by sympathetic nerves increases with the progression of diseases [39]. Central Ang-(1-7) has been reported to attenuate arterial BP regulated by sympathetic nerve activity in some cardiovascular diseases $[15,17]$. BNP-45 rises with an increase of sympathetic nerve activity. Thus, it is well reasoned that a decrease in central Ang-(1-7) is likely to lead to a higher level of BNP-45. In the current report, we specifically examined the concentrations of plasma BNP-45 in SHR and/or STZ rats and further determined if alterations of BNP-45 were closely related to the levels of Ang-(1-7) in the cerebral cortex and hippocampal CA1 area. We observed an inverse relation between BNP-45 and the levels of Ang-(1-7) in those brain areas. This further supports the notion that the levels of central Ang-(1-7) altered by cardiovascular disorders are linked to ischemic brain injuries.

Prior reports indicate that a combination of hypertension and diabetes induces a more severe cardiac functional deficit than either disease alone, which can be described as a greater reduction in cardiac performance, exacerbated fibrosis, and hypertrophy that are associated with a relatively greater increase in oxidative stress and upregulation of the renin-angiotensinogen-aldosterone system [29, 40-43]. Similar results have been observed in humans in the fact that fibrosis and cardiac hypertrophy are more pronounced in those who suffer from both hypertension and diabetes as opposed to one disorder in isolation $[44,45]$. In rat models of hypertension, it has been found that antioxidant mechanisms prevent vascular and left ventricular remodeling and dysfunction [2-5]. In diabetic rodents, antioxidant alleviates cardiac dysfunction by upregulating the NO-thioredoxin-heme oxygenase-vascular endothelial growth factor system, leading to an increase in manganese superoxide dismutase [46]. In the current study, systemic injection of tempol inhibits the levels of an oxidative stress product, 8-iso PGF2 $\alpha$, in the cerebral cortex and hippocampal CA1 region. Interestingly, we found that an increase in 8-iso PGF2 $\alpha$ is closely related to the levels of Ang-(1-7). Also, tempol can restore impaired central Ang-(1-7). The effects of tempol are greater in diabetic SHR than either SHR or STZ rats alone. This suggests that inhibition of oxidative stress has more benefits to rats with both hypertension and diabetes in improving worsen central Ang-(1-7).

Prior studies have shown that central chronic administration of Ang-(1-7) increases survival of stroke-prone SHR rats, and improves cerebral blood flow, neurological deficits and brain ischemic tolerance evoked by middle carotid artery occlusion in rats [23, 25]. These effects are not accompanied with changes of arterial BP [23,25], indicating that outcomes of Ang-(1-7) are likely independent of changes in BP. Consistent with the prior findings, in the current report, ICV injection of Ang-(1-7) fails to significantly alter BP in non-diabetic SHR animals; however, it lowers SBP in diabetic SHR animals. It should be noted that inhibitory effects of central infusion of Ang-(1-7) on BP have been reported in DOCA-salt rats [47]. The dissimilarities are likely due to hypertensive models and/or dosages of Ang-(1-7) used in the studies.

Limitations of the study need be acknowledged. First, we did not perform experiments to determine cerebrovascular functions and neurological deficit associated with ischemic brain injury though the effects of Ang-(1-7) and tempol on BP were examined. Second, in this study, we measured central Ang-(1-7) in total tissue homogenate using a competition ELISA, by which pre-purification of the sample was not required to perform. High-molecular weight proteins such as angiotensinogen were likely to be recognized by the ELISA antibody and affected assay in the present study.

\section{Conclusion}

Our findings for the first time show the levels of Ang-(1-7) and Ang-(1-7)-Mas-R-nNOS pathways in the cerebral cortex and hippocampus are downregulated to a greater degree in SHR rats with STZ injection. The changes of Ang-(1-7) are closely linked to plasma BNP. 


\section{Cellular Physiology Cell Physiol Biochem 2016;40:1186-1197 \begin{tabular}{l|l|l|}
\hline DOI: 10.1159/000453172 & $\begin{array}{l}\text { C) 2016 The Author(s). Published by S. Karger AG, Basel } \\
\text { www.karger.com/cpb }\end{array}$ \\
\hline Published ondine: Diochemistry
\end{tabular} \\ Li et al.: Central Ang 1-7 and Diabetic Hypertension}

Central injection of Ang-(1-7) improves SBP in diabetic SHR animals. This support a role for Ang-(1-7) in engagement of the adaptive mechanisms associated with diabetic hypertension. In addition, inhibition of oxidative stress is engaged in improving downregulation of Ang(1-7)-Mas-R-nNOS pathways and BP. This suggests that enhancing Ang-(1-7)-Mas-R-nNOS system is beneficial to cardiovascular and cerebrovascular dysfunction and vulnerability related to spontaneously hypertension, particularly to diabetic hypertension.

\section{Acknowledgements}

This work was supported in part by Natural Science Foundation of Heilongjiang Province (Grant\# QC2015106, Lan Zhang) and National Health and Family Planning Commission (Grant\# 2014-388, Xian Liu).

\section{Disclosure Statement}

No.

\section{References}

1 Gerstein HC, Miller ME, Byington RP, Goff DC, Jr., Bigger JT, Buse JB, Cushman WC, Genuth S, Ismail-Beigi F, Grimm RH, Jr., Probstfield JL, Simons-Morton DG, Friedewald WT: Effects of intensive glucose lowering in type 2 diabetes. N Engl J Med 2008;358:2545-2559.

2 Erejuwa 00, Sulaiman SA, Wahab MS, Sirajudeen KN, Salleh MS, Gurtu S: Differential responses to blood pressure and oxidative stress in streptozotocin-induced diabetic Wistar-Kyoto rats and spontaneously hypertensive rats: effects of antioxidant (honey) treatment. Int J Mol Sci 2011;12:1888-1907.

3 Mohamed IN, Soliman SA, Alhusban A, Matragoon S, Pillai BA, Elmarkaby AA, El-Remessy AB: Diabetes exacerbates retinal oxidative stress, inflammation, and microvascular degeneration in spontaneously hypertensive rats. Mol Vis 2012;18:1457-1466.

4 Peixoto EB, Papadimitriou A, Lopes de Faria JM, Lopes de Faria JB: Tempol reduces podocyte apoptosis via PARP signaling pathway in experimental diabetes mellitus. Nephron Exp Nephrol 2012;120:e81-90.

$5 \quad$ Vella RK, Pullen C: Resveratrol prevents cardiovascular complications in the SHR/STZ rat by reductions in oxidative stress and inflammation. Biomed Res Int 2015;2015:918123.

6 Doobay MF, Talman LS, Obr TD, Tian X, Davisson RL, Lazartigues E: Differential expression of neuronal ACE2 in transgenic mice with overexpression of the brain renin angiotensin system. Am J Physiol Regul Integr Comp Physiol 2007;292:R373-R381.

7 Santos RAS, Silva ACS, Maric C: Angiotensin-(1-7) is an endogenous ligand for the G 425 protein-coupled receptor Mas. Proc Natl Acad Sci USA 2003;100:8258-8263.

8 Yamazato M, Yamazato Y, Sun C, Diez-Freire C, Raizada MK: Overexpression of angiotensin-converting enzyme 2 in the rostral ventrolateral medulla causes long-term decrease in blood pressure in the spontaneously hypertensive rats. Hypertension 2007;49:926-931.

9 Young D, O'Neill K, Jessell T, Wigler M: Characterization of the rat mas oncogene and its high-level expression in the hippocampus and cerebral cortex of rat brain. Proc Natl Acad Sci USA 1988;85:53395342.

10 Bunnemann B, Fuxe K, Metzger R, Mullins J, Jackson TR, Hanley MR, Ganten D: Autoradiographic localization of mas proto-oncogene mRNA in adult rat brain using in situ hybridization. Neurosci Lett 1990;114:147-153.

11 Freund M, Walther T, von Bohlen und Halbach O: Immunohistochemical localization of the angiotensin-(1-7) receptor Mas in the murine forebrain. Cell Tissue Res 2012;348:29-35.

12 Martin KA, Grant SG, Hockfield S: The mas proto-oncogene is developmentally regulated in the rat central nervous system. Brain Res Dev Brain Res 1992;68:75-82. 


\section{Cellular Physiology Cell Physiol Biochem 2016;40:1186-1197 \begin{tabular}{l|l|l}
\hline DOI: 10.1159/000453172 & $\begin{array}{l}\text { @ } 2016 \text { The Author(s). Published by S. Karger AG, Basel } \\
\text { www.karger.com/cpb }\end{array}$ \\
\hline
\end{tabular} \\ Li et al.: Central Ang 1-7 and Diabetic Hypertension}

13 Metzger R, Bader M, Ludwig T, Berberich C, Bunnemann B, Ganten D: Expression of the mouse and rat mas proto-oncogene in the brain and peripheral tissues. FEBS Lett 1995;357:27-32.

14 Becker LK, Etelvino GM, Walther T, Santos RAS, Campagnole-Santos MJ: Immunofluorescence localization of the receptor Mas in cardiovascular-related areas of the rat brain. Am J Physiol Heart Circ Physiol 2007;293:H1416-H1424.

15 Ferrario CM, Trask AJ, Jessup JA: Advances in the biochemical and functional roles of angiotensin converting enzyme 2 and angiotensin-(1-7) in the regulation of cardiovascular function. . Am. J. Physiol. Heart Circ Physiol 2005; 289:H2281-H2290.

16 Campagnole-Santos MJ, Heringer SB, Batista EN, Khosla MC, Santos RA: Differential baroreceptor reflex modulation by centrally infused angiotensin peptides. Am J Physiol Heart Circ Physiol 1992;263:R89-R94.

17 Feng Y, Xia H, Cai Y, Halabi CM, Becker LK, Santos RA, Speth RC, Sigmund CD, Lazartigues E: Brain-selective overexpression of human Angiotensin-converting enzyme type 2 attenuates neurogenic hypertension. Circ Res 2010;106:373-382.

18 Mecca AP, Regenhardt RW, O'Connor TE, Joseph JP, Raizada MK, Katovich MJ, Sumners C: Cerebroprotection by angiotensin-(1-7) in endothelin-1-induced ischaemic stroke. Exp Physiol 2011;96:1084-1096.

19 Zheng JL, Li GZ, Chen SZ, Wang JJ, Olson JE, Xia HJ, Lazartigues E, Zhu YL, Chen YF: Angiotensin converting enzyme 2/Ang-(1-7)/mas axis protects brain from ischemic injury with a tendency of age-dependence. CNS Neurosci Ther 2014;20:452-459.

20 Costa MA, Verrilli MA, Gomez KA, Nakagawa P, Peña C, Arranz C, Gironacci MM: Angiotensin-(1-7) upregulates cardiac nitric oxide synthase in spontaneously hypertensive rats. Am J Physiol Heart Circ Physiol 2010;299:H1205-H1211.

21 Yang R, Yin J, Li Y, Zimmerman MC, Schultz HD: Angiotensin-(1-7) increases neuronal potassium current via a nitric oxide-dependent mechanism. Am J Physiol Cell Physiol 2011;300:C58-C64.

22 Sampson AK, Andrews KL, Graham D, McBride MW, Head GA, Thomas MC, Chin-Dusting JP, Dominiczak $\mathrm{AF}$, Jennings GL: Origin of the $\mathrm{Y}$ chromosome influences intrarenal vascular responsiveness to angiotensin I and angiotensin (1-7) in stroke-prone spontaneously hypertensive rats. Hypertension 2014;64:13761383.

23 Jiang T, Yu JT, Zhu XC, Zhang QQ, Tan MS, Cao L, Wang HF, Lu J, Gao Q, Zhang YD, Tan L: Angiotensin-(1-7) induces cerebral ischaemic tolerance by promoting brain angiogenesis in a Mas/eNOS-dependent pathway. Br J Pharmacol 2014;171:4222-4232.

24 Xu G-Y, Li G, Liu N, Huang L-Y: Mechanisms underlying purinergic P2X3 receptor mediated mechanical allodynia induced in diabetic rats. Mol Pain 2011;7:60.

25 Regenhardt RW, Mecca AP, Desland F, Ritucci-Chinni PF, Ludin JA, Greenstein D, Banuelos C, Bizon JL, Reinhard MK, Sumners C: Centrally administered angiotensin-(1-7) increases the survival of stroke-prone spontaneously hypertensive rats. Exp Physiol 2014;99:442-453.

26 Alexander CM, Landsman PB, Teutsch SM, Haffner SM: NCEP-defined metabolic syndrome, diabetes, and prevalence of coronary heart disease among NHANES III participants age 50 years and older. Diabetes 2003;52:1210-1214.

27 Kasai T, Miyauchi K, Kurata T, Okazaki S, Kajimoto K, Kubota N, Daida H: Impact of metabolic syndrome among patients with and without diabetes mellitus on long-term outcomes after percutaneous coronary intervention. Hypertens Res 2008;31:235-241.

28 Ray KK, Seshasai SR, Wijesuriya S, Sivakumaran R, Nethercott S, Preiss D, Erqou S, Sattar N: Effect of intensive control of glucose on cardiovascular outcomes and death in patients with diabetes mellitus: a meta-analysis of randomised controlled trials. Lancet 2009;373:1765-1772.

29 Lassila M, Davis BJ, Allen TJ, Burrell LM, Cooper ME, Cao Z: Cardiovascular hypertrophy in diabetic spontaneously hypertensive rats: optimizing blockade of the renin-angiotensin system. Clin Sci (Lond) 2003;104:341-347.

30 Jian K, Fok E, Cam MC, Sambandam N, Yao J, Rodrigues B: Susceptibility of spontaneously hypertensive rats to the diabetogenic effects of streptozotocin. Can J Physiol Pharmacol 1996;74:1215-1221.

31 Jiang T, Gao L, Lu J, Zhang YD: ACE2-Ang-(1-7)-Mas Axis in Brain: A Potential Target for Prevention and Treatment of Ischemic Stroke. Curr Neuropharmacol 2013;11:209-217.

32 Lovren F, Pan Y, Quan A, Teoh H, Wang G, Shukla PC, Levitt KS, Oudit GY, Al-Omran M, Stewart DJ, Slutsky AS, Peterson MD, Backx PH, Penninger JM, Verma S: Angiotensin converting enzyme-2 confers endothelial protection and attenuates atherosclerosis. Am J Physiol Heart Circ Physiol 2008;295:H1377-1384. 


\section{Cellular Physiology Cell Physiol Biochem 2016;40:1186-1197 \begin{tabular}{l|l|l} 
DOI: 10.1159/000453172 & $\begin{array}{l}\text { O 2016 The Author(s). Published by S. Karger AG, Basel } \\
\text { www.karger.com/cpb }\end{array}$
\end{tabular} \\ Li et al.: Central Ang 1-7 and Diabetic Hypertension}

33 Xia H, Sriramula S, Chhabra KH, Lazartigues E: Brain angiotensin-converting enzyme type 2 shedding contributes to the development of neurogenic hypertension. Circ Res 2013;113:1087-1096.

34 Calo LA, Schiavo S, Davis PA, Pagnin E, Mormino P, D'Angelo A, Pessina AC: ACE2 and angiotensin 1-7 are increased in a human model of cardiovascular hyporeactivity: pathophysiological implications. J Nephrol 2010;23:472-477.

35 Der Sarkissian S, Huentelman MJ, Stewart J, Katovich MJ, Raizada MK: ACE2: A novel therapeutic target for cardiovascular diseases. Prog Biophys Mol Biol 2006;91:163-198.

36 Feng Y, Xia H, Santos RA, Speth R, Lazartigues E: Angiotensin-converting enzyme 2: a new target for neurogenic hypertension. Exp Physiol 2010;95:601-606.

37 Cushman M, Judd SE, Howard VJ, Kissela B, Gutierrez OM, Jenny NS, Ahmed A, Thacker EL, Zakai NA: $\mathrm{N}$-terminal pro-B-type natriuretic peptide and stroke risk: the reasons for geographic and racial differences in stroke cohort. Stroke 2014;45:1646-1650.

38 Goya Y, Shibazaki K, Sakai K, Aoki J, Uemura J, Saji N, Isa K, Ohya Y, Kimura K: Brain natriuretic peptide upon admission as a biological marker of short-term mortality after intracerebral hemorrhage. Eur Neurol 2014;71:203-207.

39 Anand IS, Fisher LD, Chiang Y-T, Latini R, Masson S, Maggioni AP, Glazer RD, Tognoni G, Cohn JN, Investigators ftV-H: Changes in Brain Natriuretic Peptide and Norepinephrine Over Time and Mortality and Morbidity in the Valsartan Heart Failure Trial (Val-HeFT). Circulation 2003;107:1278-1283.

40 Black MJ, D'Amore A, Auden A, Stamp L, Osicka T, Panagiotopoulos S, Jerums G: Chronic type 1 diabetes in spontaneously hypertensive rats leads to exacerbated cardiac fibrosis. Cardiovasc Pathol 2010;19:361-370.

41 Chen F, Chen B, Xiao FQ, Wu YT, Wang RH, Sun ZW, Fu GS, Mou Y, Tao W, Hu XS, Hu SJ: Autophagy protects against senescence and apoptosis via the RAS-mitochondria in high-glucose-induced endothelial cells. Cell Physiol Biochem 2014;33:1058-1074.

42 Raza H, John A, Howarth FC: Increased oxidative stress and mitochondrial dysfunction in zucker diabetic rat liver and brain. Cell Physiol Biochem 2015;35:1241-1251.

43 Wold LE, Relling DP, Colligan PB, Scott GI, Hintz KK, Ren BH, Epstein PN, Ren J: Characterization of contractile function in diabetic hypertensive cardiomyopathy in adult rat ventricular myocytes. J Mol Cell Cardiol 2001;33:1719-1726.

44 Grundy SM: Metabolic syndrome update. Trends Cardiovasc Med 2016;26:364-373.

45 Kintscher U: Benefit of Blood Pressure Control in Diabetic Patients. Curr Hypertens Rep 2015;17:50.

46 Thirunavukkarasu M, Penumathsa SV, Koneru S, Juhasz B, Zhan L, Otani H, Bagchi D, Das DK, Maulik $\mathrm{N}$ : Resveratrol alleviates cardiac dysfunction in streptozotocin-induced diabetes: Role of nitric oxide, thioredoxin, and heme oxygenase. Free Radic Biol Med 2007;43:720-729.

47 Guimaraes PS, Santiago NM, Xavier CH, Velloso EP, Fontes MA, Santos RA, Campagnole-Santos MJ: Chronic infusion of angiotensin-(1-7) into the lateral ventricle of the brain attenuates hypertension in DOCA-salt rats. Am J Physiol Heart Circ Physiol 2012;303:H393-400. 